\title{
Optimal Trajectory of Underwater Manipulator Using Adjoint Variable Method for Reducing Drag
}

\author{
Kazunori Shinohara \\ JAXA'S Engineering Digital Innovation Center, Japan Aerospace Exploration Agency, Sagamihara City, Japan \\ E-mail: shinohara@06.alumni.u-tokyo.ac.jp \\ Received June 28, 2011; revised July 30, 2011; accepted August 16, 2011
}

\begin{abstract}
In order to decrease the fluid drag on an underwater robot manipulator, an optimal trajectory method based on the variational method is presented. By introducing the adjoint variables, which are Lagrange multipliers, we formulate a Lagrange function under certain constraints related to the target angle, target angular velocity, and dynamic equation of the robot manipulator. The state equation (the partial differentiation of the Lagrange function with respect to the state variables), adjoint equation (the partial differentiation of the Lagrange function with respect to the adjoint variables), and sensitivity equation (the partial differentiation of the Lagrange function with respect to torques) can be derived from the stationary conditions of the Lagrange function. Using the state equation, we can calculate the state variables (angles, angular velocities, and angular acceleration) at every time step in the forward time direction. These state variables are stored as data at every time step. Next, by using the adjoint equation, we can calculate the adjoint variables by using these state variables at every time step in the backward time direction. These adjoint variables are stored as data at every time step. Third, the sensitivity equation is calculated by using both the state variables and the adjoint variables. Finally, the optimal trajectory of the manipulator is obtained using the sensitivities. The proposed method is applied to the problem of two-link manipulators. It can obtain the optimal drag reduction trajectory of the manipulator under the constraints mentioned above.
\end{abstract}

Keywords: Robot Manipulator Dynamics, Optimal Trajectory, Adjoint Variable Method, Euler-Lagrange Equation, Fluid Drag Force, Calculus of Variations

\section{Introduction}

Presently, we are facing serious environmental problems such as global warming and abnormal climatic conditions, which are closely related to the ocean. Therefore, the establishment of ocean study technology is extremely important. Since the 1990s, researchers have investigated the development of underwater robot manipulators for oceanic studies [1-6].

In an extreme environment such as the abyssal ocean, it is difficult to supply energy to manipulators. However, because of fluid tractions, the energy consumption of an underwater manipulator is greater than that of a manipulator in air. In order to reduce the energy consumption, it is important to determine the optimal trajectory to reduce the drag on the manipulator.

Optimal time control for a manipulator trajectory was studied in the 1970s [7,8]. Kahn and Roth first presented an optimal time control method based on kinematic dynamics [9]. Vukobratovic and Kiranski presented an optimal time control method based on dynamic programming [10]. Townsend et al. presented optimal control by approximating a function [11]. Lee et al. presented the formulation of a genetic algorithm based on trajectory planning [12]. Constantinescu et al. presented a method for determining smooth and time-constrained optimal path trajectories for a robot manipulator [13]. These studies were carried out with the objective of constructing an optimal time trajectory for a manipulator, from its initial position to the target position. On the other hand, Eiji presented a method for determining the minimum energy trajectory of an underwater manipulator [14]. Uno et al. presented a minimum torque change model [15]

A method for drag reduction control has not been developed thus far. A marine robot has energy limitations during its operations. Therefore, drag reduction control 
under an extreme environment is crucial for low energy consumption.

In this study, we propose an optimal trajectory method for reducing the drag on the manipulator. As the manipulator moves from its initial position to the target position, the fluid generates an external force on the manipulator. A method based on the variational principle is developed to determine the optimal trajectory to reduce the drag. This method is called the adjoint variable method. The adjoint variable method is based on a variational method. By introducing Lagrange multipliers called adjoint variables, we transform the constrained optimization of the cost function into the unconstrained optimization of the Lagrange function. The cost function is defined as the fluid drag on the manipulator. The Lagrange function is formulated under the constraints of the robot manipulator dynamics. The stationary conditions (the state equation, adjoint equation, and sensitivity equation) are derived from the Lagrange function. An algorithm is developed on the basis of the stationary conditions. First, the state variables (the angle, angular velocity, and angular accelerations) are calculated by using the state equation in the forward time direction and stored as data at every time step. Next, by using the state variables at every time step, we calculate the adjoint variables by using the adjoint equation in the backward time direction. Finally, the sensitivity (gradient) is calculated at every time step, and the time history of the joint torques is determined.

Using this optimal trajectory algorithm developed in three phases (state analysis, adjoint analysis, and sensitivity analysis), we resolve the problem of the two-link manipulator. The effectiveness of the algorithm is then verified by comparing it with the optimal time control methods described in the literature.

\section{Theory}

\subsection{Variable}

In this paper, the two-dimensional motion of a manipulator with respect to the $x-y$ plane is considered, as shown in the Figure 1. The links are arranged in the shape of a circular cylinder. The manipulator consists of two links that are connected by joints. The coordinates at each joint are defined. The joints and links are numbered from the base to the tip.

The angles with respect to joint $i$ are defined as

$$
q_{\mathrm{i}} i=1,2
$$

The angular velocities with respect to joint $i$ are defined as:

$$
\dot{q}_{\mathrm{i}} i=1,2
$$

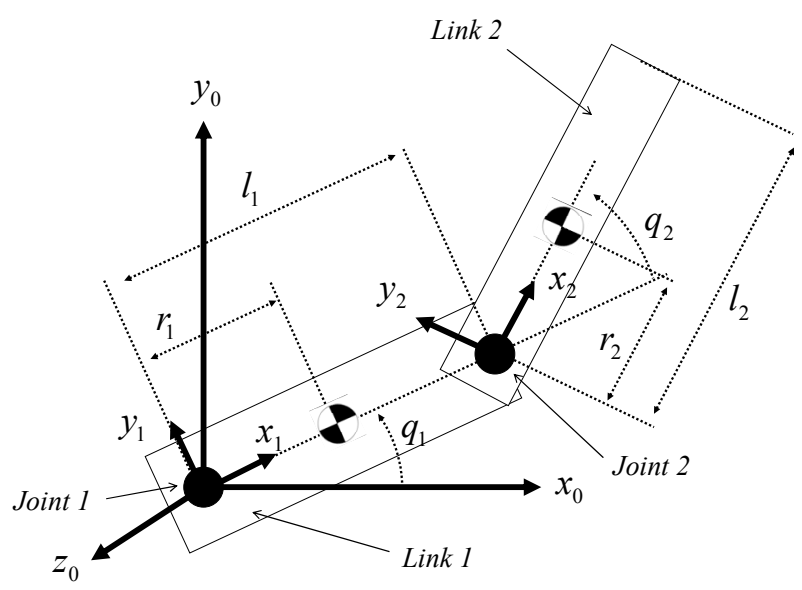

Figure 1. Two-link manipulator.

The angular accelerations with respect to joint $i$ are defined as

$$
\ddot{q}_{\mathbf{i}} i=1,2
$$

In this study, the variables obtained from Eq.(1) to Equation (3) are called state variables $\left(\boldsymbol{q}=\left(q_{1}, q_{2}, \dot{q}_{1}, \dot{q}_{2}\right.\right.$, $\left.\ddot{q}_{1}, \ddot{q}_{2}\right)$ ). The penalty parameters with respect to the angle and angular velocity are defined as

$$
\varepsilon_{\mathbf{i}} i=1,2,3,4
$$

Variables with respect to the angular accelerations $\left(\ddot{q}_{1}, \ddot{q}_{2}\right)$ are defined as

$$
\lambda_{\mathrm{i}} i=1,2
$$

The variables obtained from Equation (5) are called adjoint variables $\left(\lambda=\left(\lambda_{1}, \lambda_{2}\right)\right)$. The torques with respect to joint $i$ are defined as

$$
\tau_{\mathbf{i}} i=1,2
$$

The masses with respect to link $i$ are defined as

$$
m_{\mathbf{i}} i=1,2
$$

The diameters with respect to link $i$ are defined as

$$
d_{\mathbf{i}} i=1,2
$$

The lengths with respect to link $i$ are defined as

$$
l_{\mathbf{i}} i=1,2
$$

The lengths from the centroid of a link to joint $i$ are defined as

$$
r_{\mathbf{i}} i=1,2
$$

The drag coefficients with respect to link $i$ are defined as

$$
C_{\mathbf{i}} i=1,2
$$

The density of the water is defined as

$$
\rho
$$


The translational velocities with respect to link $i$ are defined as

$$
v_{\mathbf{i}} i=1,2
$$

The sign function $\operatorname{sgn}(x)$ is defined as

$$
\operatorname{sgn}(x)=\left\{\begin{array}{lll}
-1 & \text { if } & x<0 \\
0 & \text { if } & x=0 \\
1 & \text { if } & x>0
\end{array}\right.
$$

In order to derive the dynamics of an underwater robot manipulator, the external force exerted by the fluid drag needs to be added to the robot manipulator dynamics. The fluid drag on an object is proportional to the square of the object's speed [16]. The fluid drag always has a positive value in the calculation of the robot manipulator dynamics. With respect to the motion direction of the manipulator, the fluid drag acts in the opposite direction in a real environment. Therefore, the sign of the fluid drag direction has to be determined according to the motion of the manipulator. The motion direction of the manipulator can be identified by the sign of the translational velocities.

\subsection{Problem}

In order to minimize the cost function under certain constraints, a Lagrange function is formulated by introducing the adjoint variables. The input data are the time histories of the torques $\left(\tau_{1}, \tau_{2}\right)$ from the start time 0 to the end time $t$. The optimal trajectory is searched for in the set of inputs. After determining the values of $\tau_{1}$ and $\tau_{2}$, the angle, angular velocities, and angular accelerations are determined using the state equation from start time 0 to the end time $t$. The tip of the underwater manipulator moves from the initial position to the target position. The objective of this study is to reduce the fluid drag by the $2 \mathrm{D}$ trajectory of the manipulator. The cost function is defined as

$$
\begin{aligned}
J= & D_{1}+D_{2}+N \\
N= & \varepsilon_{1}\left(q_{1}-a\right)^{2}+\varepsilon_{2}\left(q_{2}-b\right)^{2}+\varepsilon_{3}\left(\dot{q}_{1}-c\right)^{2}+\varepsilon_{4}\left(\dot{q}_{2}-d\right)^{2} \\
= & \varepsilon_{1}\left(\int_{0}^{t} \dot{q}_{1} \mathrm{~d} t-q_{1}(0)-a\right)\left(q_{1}-a\right) \\
& +\varepsilon_{2}\left(\int_{0}^{t} \dot{q}_{2} \mathrm{~d} t-q_{2}(0)-b\right)\left(q_{2}-b\right) \\
& +\varepsilon_{3}\left(\dot{q}_{1}-c\right)^{2}+\varepsilon_{4}\left(\dot{q}_{2}-d\right)^{2} \\
& D_{1}=\frac{\rho}{6} C_{1} d_{1} l_{1}^{3} \dot{q}_{1}^{2} \cdot \operatorname{sgn}\left(\dot{q}_{1}\right)
\end{aligned}
$$

$$
\begin{gathered}
D_{2}=\frac{\rho}{2} C_{2} d_{2} l_{2}\left\{\left(l_{1}^{2}+l_{1} l_{2}+\frac{l_{2}^{2}}{3}\right) \dot{q}_{1}^{2}+\left(\frac{2 l_{2}^{2}}{3}+l_{1} l_{2}\right) \dot{q}_{1} \dot{q}_{2}\right. \\
\left.+\frac{l_{2}^{2}}{3} \dot{q}_{2}^{2}\right\}
\end{gathered}
$$

where the parameters $a, b, c$, and $d$ in Equation (16) are constants. The first and second terms in Equation (16) are the constraints with respect to the target angle $a$ of joint 1 and target angle $b$ of joint 2, respectively. The third and fourth terms in Equation (16) are the constraints with respect to the target angular velocity $c$ of joint 1 and target angular velocity $d$ of joint 2 , respectively. Equations (17) and (18) represent the fluid drag on link 1 and link 2, respectively (see Appendix A). In order to simplify the formulation of the adjoint variable method in this study, it is assumed that the inequality $l_{1} \dot{q}_{1}+l_{2}\left(\dot{q}_{1}+\dot{q}_{2}\right)>0$ is satisfied at all times. The Lagrange function is defined as

$$
L=J+\lambda F=D_{1}+D_{2}+N+\lambda_{1} F_{1}+\lambda_{2} F_{2}
$$

where equations $F_{1}$ and $F_{2}$ constitute the state equation. This state equation represents the robot manipulator dynamics. In this study, a weak formulation is applied to the Lagrange function by the time integration of the state equation. The Lagrange function can also be formulated by a strong formulation that satisfies the equation at every time step.

\subsection{State Equation}

The partial differential equation of the Lagrange function with respect to the adjoint variable $\lambda$ is called the state equation:

$$
\begin{aligned}
& \frac{\partial L}{\partial \lambda_{1}}-\frac{\mathrm{d}}{\mathrm{d} t}\left(\frac{\partial L}{\partial \dot{\lambda}_{1}}\right)=F_{1}=0 \quad\left(\frac{\partial L}{\partial \dot{\lambda}_{1}}=0\right) \\
& \frac{\partial L}{\partial \lambda_{2}}-\frac{\mathrm{d}}{\mathrm{d} t}\left(\frac{\partial L}{\partial \dot{\lambda}_{2}}\right)=F_{2}=0 \quad\left(\frac{\partial L}{\partial \dot{\lambda}_{2}}=0\right)
\end{aligned}
$$

Equations (20)-(21) represent the robot dynamic manipulator as

$$
M(q) \ddot{q}+C(q, \dot{q})+f(\dot{q})=\tau
$$

The parameters $M, C, f$, and $\tau$ represent the inertia matrix, vector of the coriolis and centrifugal forces, drag force, and joint torque, respectively. By using the parameters in Appendix B, Eqaution (22) is written as

$$
\left[\begin{array}{cc}
A_{6} & -A_{2} \\
-A_{2} & B_{2}
\end{array}\right]\left[\begin{array}{l}
\ddot{q}_{1} \\
\ddot{q}_{2}
\end{array}\right]-\left[\begin{array}{c}
A_{5} \\
A_{3}
\end{array}\right]-\left[\begin{array}{l}
A_{13} \\
A_{14}
\end{array}\right]=\left[\begin{array}{l}
\tau_{1} \\
\tau_{2}
\end{array}\right]
$$

Equation $F$ is defined as 


$$
\begin{aligned}
{\left[\begin{array}{l}
F_{1} \\
F_{2}
\end{array}\right]=} & -\left[\begin{array}{l}
\ddot{q}_{1} \\
\ddot{q}_{2}
\end{array}\right]+\left[\begin{array}{c}
\frac{A_{2} A_{3}+B_{2} A_{5}}{A_{1}} \\
\frac{A_{2} A_{5}+A_{3} A_{6}}{A_{1}}
\end{array}\right]+\left[\begin{array}{c}
\frac{A_{2} A_{14}+B_{2} A_{13}}{A_{1}} \\
\frac{A_{2} A_{13}+A_{6} A_{14}}{A_{1}}
\end{array}\right] \\
& +\left[\begin{array}{ll}
\frac{B_{2}}{A_{1}} & \frac{A_{2}}{A_{1}} \\
\frac{A_{2}}{A_{1}} & \frac{A_{6}}{A_{1}}
\end{array}\right]\left[\begin{array}{l}
\tau_{1} \\
\tau_{2}
\end{array}\right]=\left[\begin{array}{l}
0 \\
0
\end{array}\right]
\end{aligned}
$$

where parameters $A_{1}-A_{14}, B_{1}$, and $B_{2}$ are discussed in Appendix $B$ and Appendix $C$.

\subsection{Adjoint Equations}

The adjoint equations are the Euler-Lagrange equations derived from the stationary condition of the Lagrange function. The adjoint equations are derived as

$$
\begin{aligned}
& \frac{\partial L}{\partial \dot{q}_{1}}-\frac{\mathrm{d}}{\mathrm{d} t}\left(\frac{\partial L}{\partial \ddot{q}_{1}}\right)=0 \\
& \frac{\partial L}{\partial \dot{q}_{2}}-\frac{\mathrm{d}}{\mathrm{d} t}\left(\frac{\partial L}{\partial \ddot{q}_{2}}\right)=0
\end{aligned}
$$

The time derivation of the adjoint variable $\lambda_{1}$ is derived from Equation (25) (see Appendix $D$ ).

$$
\begin{aligned}
\dot{\lambda}_{1}= & -\varepsilon_{1} t\left(q_{1}-a\right)-2 \varepsilon_{3}\left(\dot{q}_{1}-c\right)+2 B_{4} \dot{q}_{1}+B_{5} A_{16} \\
& -\lambda_{1}\left(\frac{-2 A_{2} A_{10}+B_{2} A_{7}}{A_{1}}+\frac{A_{2} B_{5} r_{2} A_{16}+B_{2} A_{17}}{A_{1}}\right) \\
& -\lambda_{2}\left(\frac{A_{2} A_{7}-2 A_{10} A_{6}}{A_{1}}+\frac{A_{2} A_{17}+A_{6} B_{5} r_{2} A_{16}}{A_{1}}\right)
\end{aligned}
$$

The time derivation of the adjoint variable $\lambda_{2}$ is derived from Equaiton (26).

$$
\begin{aligned}
\dot{\lambda}_{2}= & -\varepsilon_{2} t\left(q_{2}-b\right)-2 \varepsilon_{4}\left(\dot{q}_{2}-d\right)+B_{5} A_{18} \\
& -\lambda_{1}\left(\frac{B_{2} A_{12}+A_{2} B_{5} r_{2} A_{18}+B_{2} A_{18} A_{19}}{A_{1}}\right) \\
& -\lambda_{2}\left(\frac{A_{2} A_{12}+A_{2} A_{18} A_{19}+A_{6} B_{5} r_{2} A_{18}}{A_{1}}\right)
\end{aligned}
$$

The condition of the end time $t_{f}$ is derived from the partial differential equation of the Lagrange function with respect to the state variables as

$$
\frac{\partial L\left(t_{f}\right)}{\partial \ddot{q}_{1}}=0, \quad \frac{\partial L\left(t_{f}\right)}{\partial \ddot{q}_{2}}=0
$$

Using Equation (29), we obtain the following equations:

$$
\lambda_{i}\left(t_{f}\right)=0 \quad i=1,2
$$

\subsection{Sensitivity Equations}

The partial differential equation of the Lagrange function with respect to the torque $\tau$ is called the sensitivity equation:

$$
\frac{\partial L}{\partial \tau_{1}}-\frac{\mathrm{d}}{\mathrm{d} t}\left(\frac{\partial L}{\partial \dot{\tau}_{1}}\right)=\frac{\lambda_{1} B_{2}+\lambda_{2} A_{2}}{A_{1}}=G_{1,(k)}(t)=0 \quad\left(\frac{\partial L}{\partial \dot{\tau}_{1}}=0\right)
$$

$$
\frac{\partial L}{\partial \tau_{2}}-\frac{\mathrm{d}}{\mathrm{d} t}\left(\frac{\partial L}{\partial \dot{\tau}_{2}}\right)=\frac{\lambda_{1} A_{2}+\lambda_{2} A_{6}}{A_{1}}=G_{2,(k)}(t)=0 \quad\left(\frac{\partial L}{\partial \dot{\tau}_{2}}=0\right)
$$

where the subscript $(k)$ represents an iteration, as shown in the Figure 2. The time histories of the torques are iteratively modified from the time histories of the initial torques. The algorithm determines the optimal trajectory by minimizing the Lagrange function. Finally, $G_{i,(k)}(t)(I$ $=1,2)$ reaches zero if the subscript $(k)$ represents a sufficient number of iterations.

\subsection{Steepest Descent Method}

The time histories of the torques are modified by the gradient as

$$
\begin{gathered}
\tau_{1,(k+1)}(t)=\tau_{1,(k)}(t)+\alpha G_{1,(k)}(t) \\
\tau_{2,(k+1)}(t)=\tau_{2,(k)}(t)+\alpha G_{2,(k)}(t)
\end{gathered}
$$

The value of the coefficient $\alpha$ should be sufficiently small in order to robustly converge to the optimal trajectory and to avoid numerical vibration and divergence. In this study, the parameter $\alpha$ is set to 0.1 .

\section{Algorithm}

The algorithm is shown in the Figure 2. In the first phase, the state variable $(q)$ is calculated from the start time to the end time in the forward direction. The data of the state variable at every time step are stored in the $P C$ memory. In the second phase, using the state variable at every time step, we can calculate the adjoint variables $(\lambda)$ from the end time to the start time in the backward direction. The adjoint variables are also stored at every time step. In the third phase, by using the state variable and adjoint variable, we obtain sensitivity $G$, which is the gradient of the Lagrange function, at every time step. The time histories of the torques are modified by using the steepest descent method. Finally, the results are visualized in the case where the position of the manipulator 


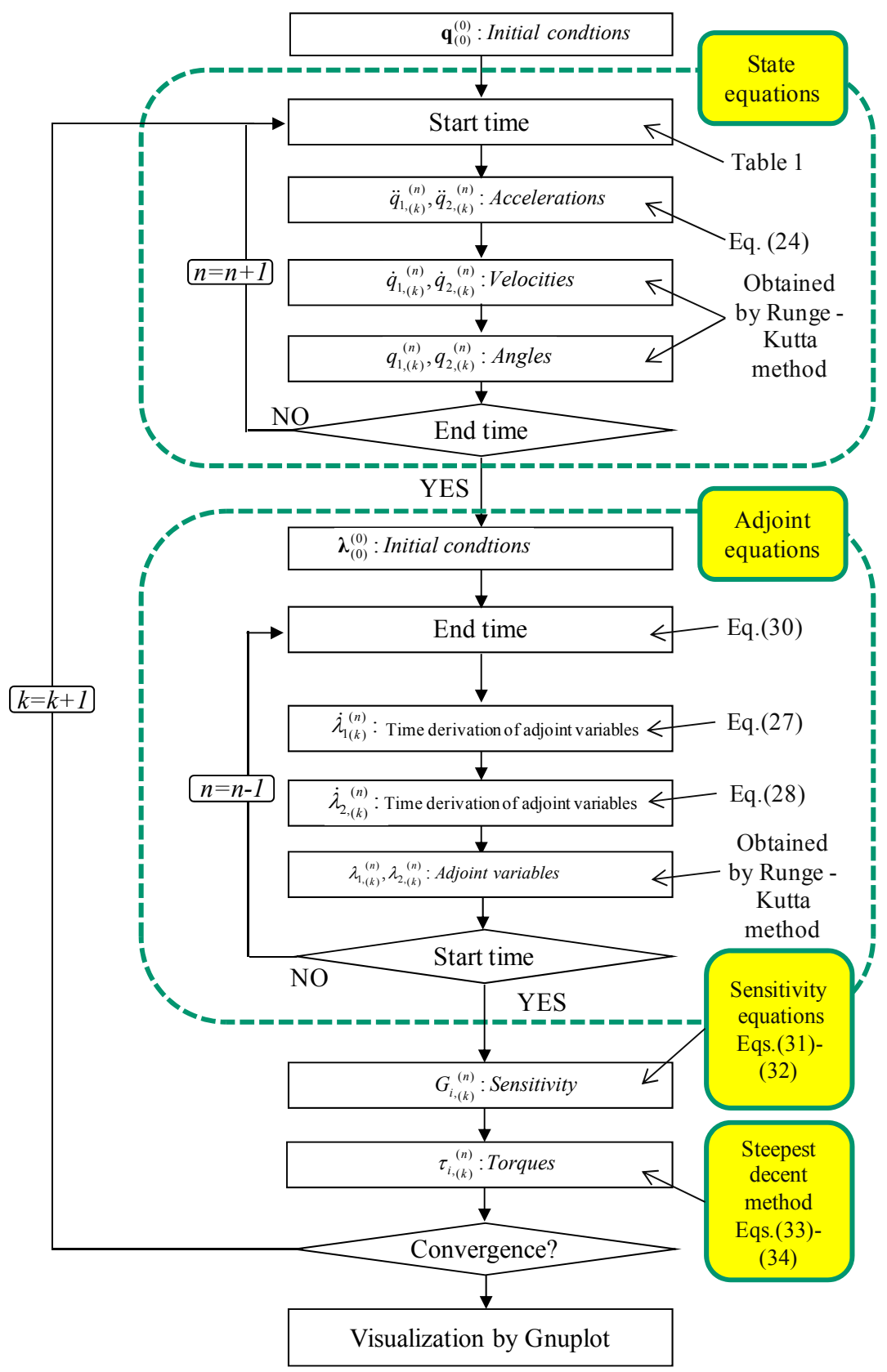

Figure 2. Algorithm.

almost agrees with the target position. In the case where the position of the manipulator does not reach the target position, this algorithm returns to the first phase.

\section{Results}

In Section 2, we formulated the adjoint variable method for reducing the drag on a manipulator. Using this method, the drag reduction trajectory can be obtained. The optimal trajectory obtained by the drag reduction control method can be verified by comparing it with the optimal trajectory obtained by the time optimal control method described in the literature [17] (see Appendix E). Using the time optimal control method, the manipulator requires a minimum amount of time to move from the initial position to the target position.

\subsection{Calculation Conditions}

Two trajectories for manipulators located at different initial positions are calculated using both the time optimal control and the drag reduction control. These two 
calculation conditions are defined as case 1 and case 2 . The initial and objective positions are summarized in Table 1.

The edge of link 1 is fixed at the origin $(0,0)$. The maximum and minimum values for both torque 1 and torque 2 are set to $\pm 30(N)$ and $\pm 10(N)$, respectively. The initial parameters are summarized in Table 1. The solver is applied to the Runge-Kutta method. The time span is set to 0.001 . The time histories of the initial torques, $\tau_{i,(k)}$ $(I=1,2)$, are constantly defined as zero. The parameters $B_{1}=4.5337, B_{2}=0.3033$, and $B_{3}=0.1482$ in the literature [17] are used in the manipulator computing model (see Appendix B). The density $\rho$ is set to 1.0. The drag coefficients, $C_{1}$ in link 1 and $C_{2}$ in link 2, with respect to the circular cylinder are each set to 0.1 . The lengths, $L_{1}$ of link 1 and $L_{2}$ of link 2, are each set to 1.0. The diameters of the cylinder, $d_{l}$ and $d_{2}$, with respect to link 1 and link 2 are each set to 0.01 . The penalty parameters, $\varepsilon_{1} \sim \varepsilon_{4}$, are set to 5 .

\subsection{Computational Results in Case 1}

The trajectories for case 1 are shown in the Figure 3 (time optimal control) and the Figure 4 (drag reduction control). The end time is 0.81 . The time histories of the angles are shown in the Figure 5. The black and red lines in the figures show angle 1 and angle 2, respectively. Angle $1\left(q_{1}\right)$ constantly converges to the target angle. During the first $0.4 \mathrm{~s}$, angle $2\left(q_{2}\right)$ is away from the target angle. After that, by rapidly closing to the target angle, the time optimal trajectory using the inertia force is created. As shown in the Figure 7, the angular velocities $\left(\dot{q}_{1}, \dot{q}_{2}\right)$ almost become zero at end time $t_{f}$ and satisfy the constraint condition. In link 1 of the manipulator, the angular velocity $\dot{q}_{1}$ increases monotonically during approximately the first $0.4 \mathrm{~s}$. After that, the angular velocity $\dot{q}_{1}$ decreases monotonically in order to satisfy $\dot{q}_{1}=0$. In link 2 of the manipulator, the angular velocity $\dot{q}_{2}<0$ from 0 s to $0.4 \mathrm{~s}$. After that, the angular

Table 1. Initial and target conditions in case 1 and case 2.

\begin{tabular}{cccc}
\hline & Parameter & Initial condition & Target condition \\
\cline { 2 - 4 } Case 1 & $q_{1}$ & $-\pi / 3$ & 0.0 \\
& $q_{2}$ & 0.0 & 0.0 \\
& $\dot{q}_{1}$ & 0.0 & 0.0 \\
& $\dot{q}_{2}$ & 0.0 & 0.0 \\
Case 2 & $q_{1}$ & $\pi / 3$ & 0.0 \\
& $q_{2}$ & $-\pi / 6 \times 5$ & 0.0 \\
& $\dot{q}_{1}$ & 0.0 & 0.0 \\
\hline
\end{tabular}

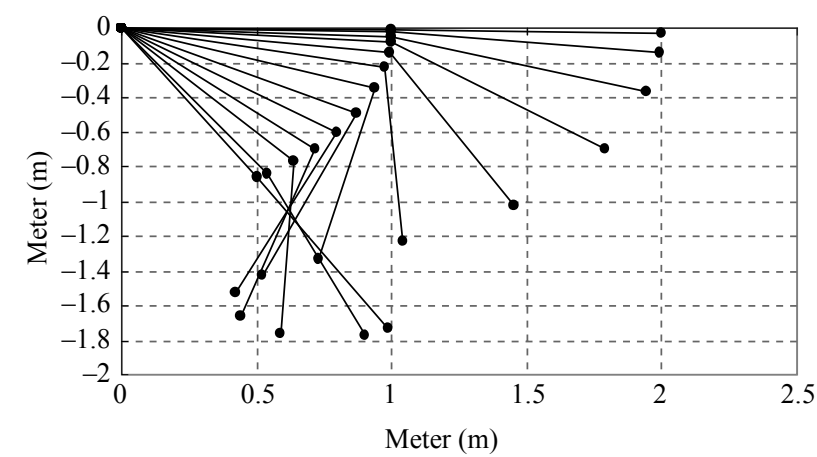

Figure 3. Trajectory of two-link manipulator for time optimal control in case 1.

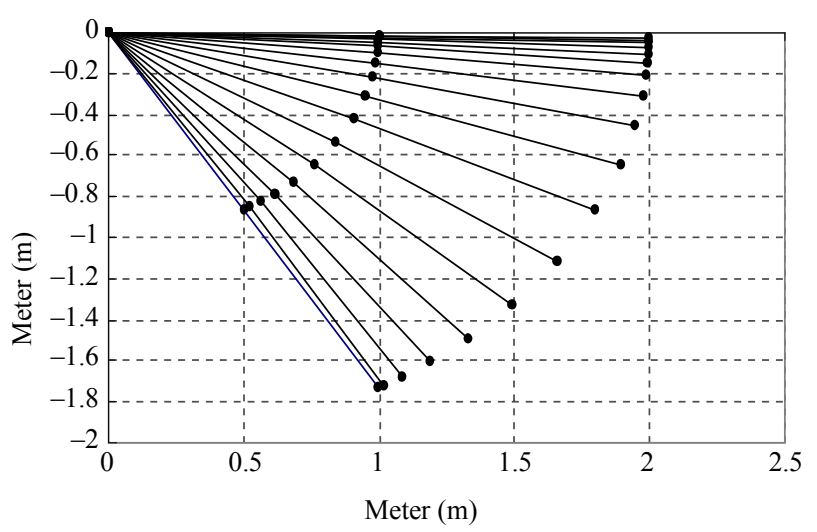

Figure 4. Trajectory of two-link manipulator for drag reduction control in case 1 .

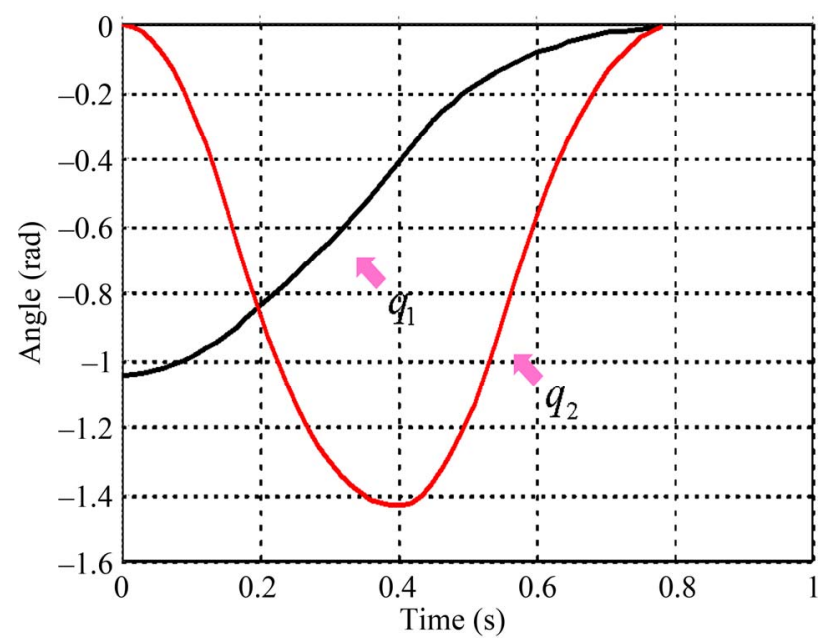

Figure 5. Time history of angles for time optimal control in case 1.

velocity $\dot{q}_{2}>0$, and it decreases in order to satisfy the constraint, $\dot{q}_{2}=0$ at end time $t_{f}$. The time history of the torques for the time optimal control is shown in the Figure 9. The maximum torque of $+30(\mathrm{Nm})$ acts on joint 1 during approximately the first $0.4 \mathrm{~s}$. After that, in order 
to meet the end constraint condition, $\dot{q}_{1}=0$, the inverse maximum torque of $-30(\mathrm{Nm})$ acts on joint 1 . The maximum torque of $-10(\mathrm{Nm})$ acts on joint 2 from $0.0 \mathrm{~s}$ to $0.15 \mathrm{~s}$. After that, the inverse maximum torque of +10 $(\mathrm{Nm})$ acts on joint 2 during $0.19 s-0.50 \mathrm{~s}$. Again, the maximum torque of $-10(\mathrm{Nm})$ acts on joint 2 .

The drag reduction trajectory and the time history of angles are shown in Figure 4 and Figure 6, respectively. The end time $t_{f}$ is $1.08 \mathrm{~s}$. The angle $q_{2}$ has a constant value of zero. By avoiding any extra movement of the manipulator, a trajectory from the initial position to the end position is created for minimum drag reduction.

The time history of the angular velocity is shown in the Figure 8. Equaiton (40) is satisfied. The angular velocity $\dot{q}_{1}$ increases monotonically during approximately the first $0.4 s$. After that, in order to satisfy the constraint condition, $\dot{q}_{1}=0$, angular velocity $\dot{q}_{1}$ decreases monotonically. The angular velocity $\dot{q}_{2}$ remains close to zero by adjusting torque 2 at joint 2 .

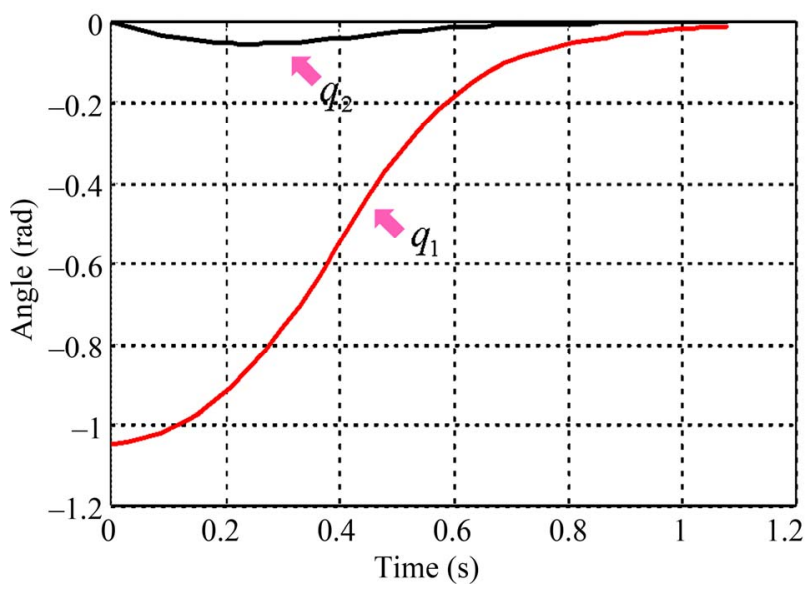

Figure 6. Time history of angles for drag reduction control in case 1.

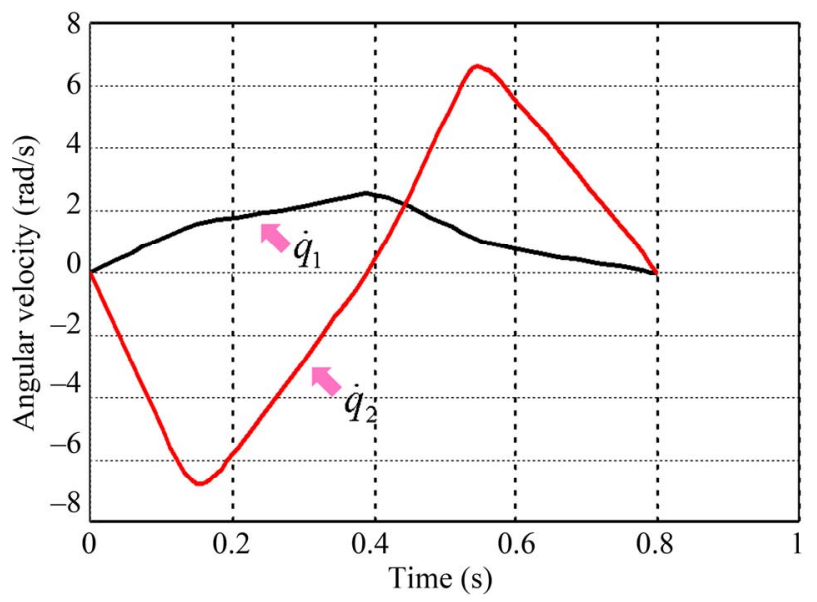

Figure 7. Time history of angular velocities for time optimal control in case 1.

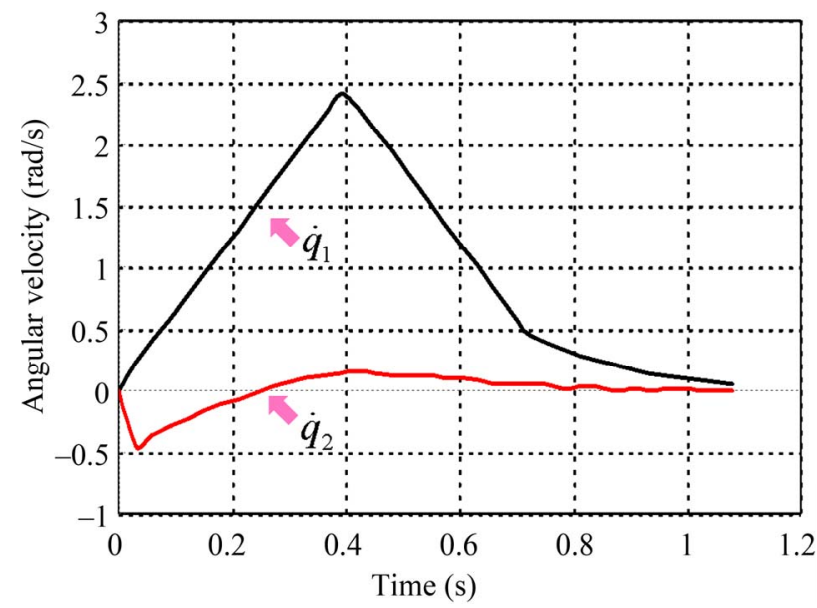

Figure 8. Time history of angular velocities for drag reduction control in case 1 .

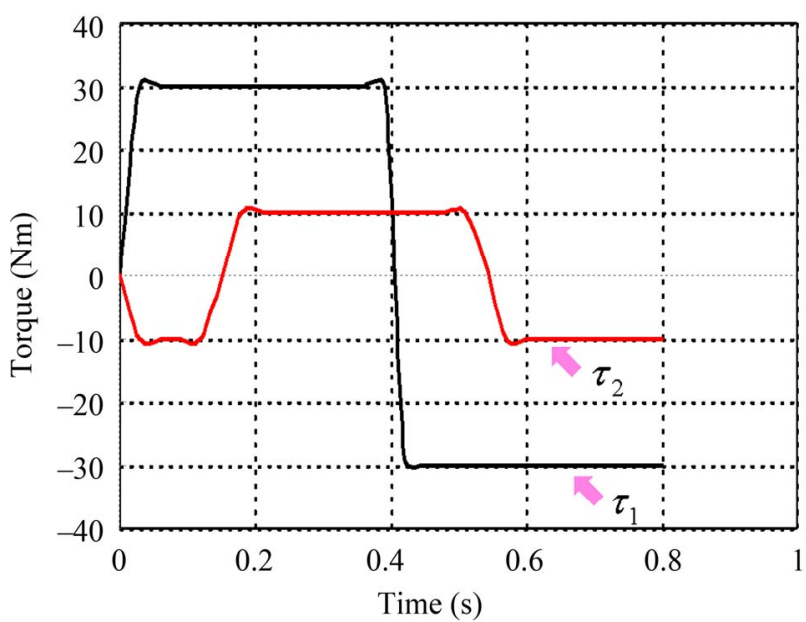

Figure 9. Time history of torques for time optimal control in case 1.

The time history of the torques for the drag reduction control is shown in Figure 10. The maximum torque of $+30(\mathrm{Nm})$ acts on joint 1 from $0.0 \mathrm{~s}$ to $0.4 \mathrm{~s}$. After that, by loading the inverse torque, torque 1 is adjusted such that $\dot{q}_{1}=0$. In the case of torque 2 , in order to satisfy $q_{2}=0$ and $\dot{q}_{2}=0$, alternative torques using both the positive torque and the negative torque act on joint 2 .

\subsection{Computational Results in Case 2}

For case 2, as shown in Table 1, the trajectory of the manipulator obtained by the time optimal control is shown in the Figure 11. The end time is $0.71 \mathrm{~s}$. The time histories of the angles are shown in the Figure 13. The angle $q_{1}$ constantly converges to the target angle. During the first $0.2 s$, angle $2\left(q_{2}\right)$ is away from the target angle. After that, by rapidly closing to the target angle, a time optimal trajectory is created using the pullback force 
obtained by the inertia force.

The time histories of the angular velocities are shown in the Figure 15. In link 1, the absolute value of the angular velocity $\dot{q}_{1}$ increases monotonically during approximately the first $0.35 \mathrm{~s}$.

After that, the absolute value of angular velocity $\dot{q}_{1}$ decreases monotonically in order to satisfy $\dot{q}_{1}=0$. Angular velocity $\dot{q}_{2}$ becomes $\dot{q}_{2}<0$ during the first 0.18 $s$. After that, parameter $\dot{q}_{2}$ turns to $\dot{q}_{2}>0$.

The time histories of the torques for the time-optimal trajectory are shown in the Figure 17. The maximum torques of $+30(\mathrm{Nm})$ act on joint 1 during approximately the first $0.35 s$. After that, the inverse torques act on joint 1 so that $\dot{q}_{1}=0$.

The clockwise torque 2 acts on joint 2 during the first $0.1 s$. The counterclockwise torque takes effect from 0.1 $s$ to $0.45 s$. Again, the clockwise torque takes effect after $0.45 s$

For case 2, as shown in Table 1, the trajectory of the manipulator obtained by the drag reduction control and the time history of angles for drag reduction control in case 2 are shown in the Figure 12 and the Figure 14, respectively. The end time is $1.08 \mathrm{~s}$. In order to reduce the cost function, a small angle for $q_{2}$ is selected at every time step. To prevent the generation of drag by any extra motion of the manipulator, the manipulator is prevented from swinging link 2 with respect to the target angle. Link 2 of the manipulator moves in a straight path with respect to the target position.

The time histories of the angular velocities are shown in the Figure 16. Equation (40) is satisfied. The absolute value of the velocities, $\dot{q}_{1}$, increases monotonically. After that, the absolute value of the velocities, $\dot{q}_{1}$, decreases monotonically in order to satisfy $\dot{q}_{1}=0$. Angular velocity $\dot{q}_{2}$ increases monotonically. After that, $\dot{q}_{2}$ decreases monotonically in order to satisfy $\dot{q}_{2}=0$.

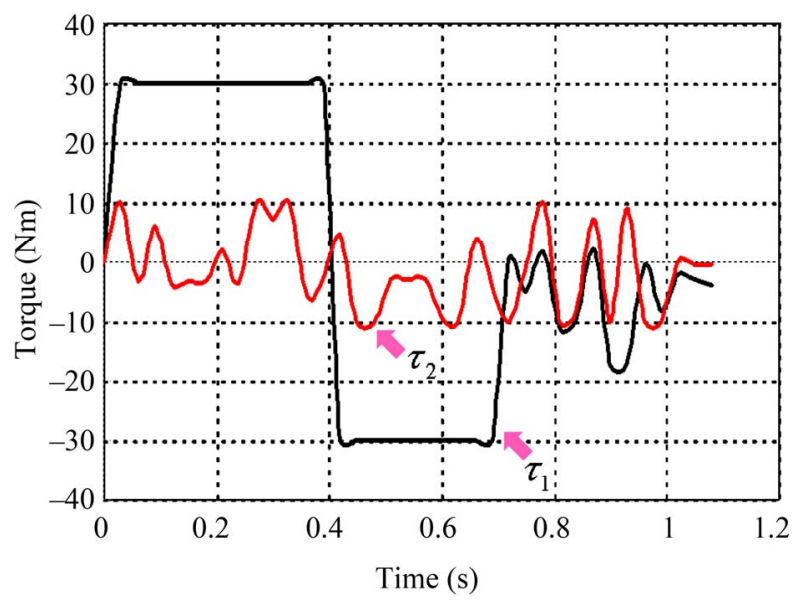

Figure 10. Time history of torques for drag reduction control in case 1.

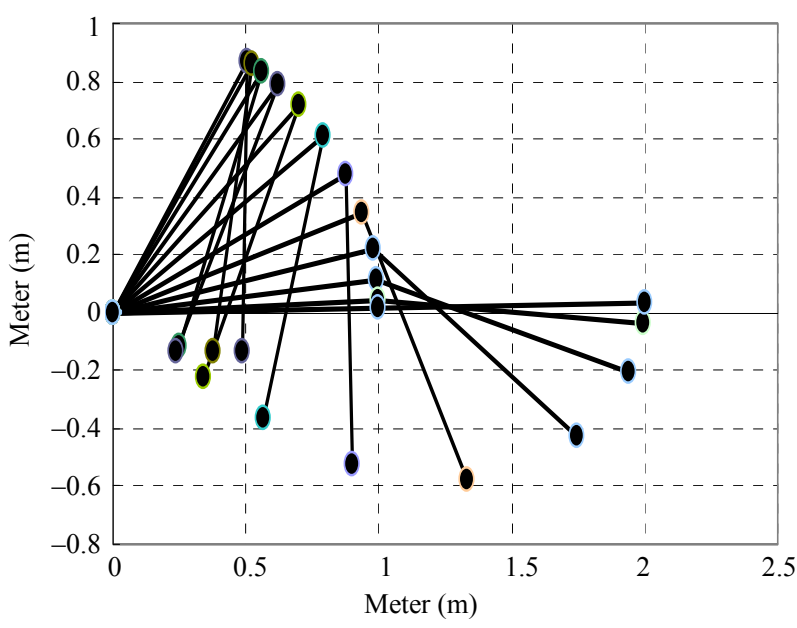

Figure 11. Trajectory of two-link manipulator for time optimal control in case 2 .

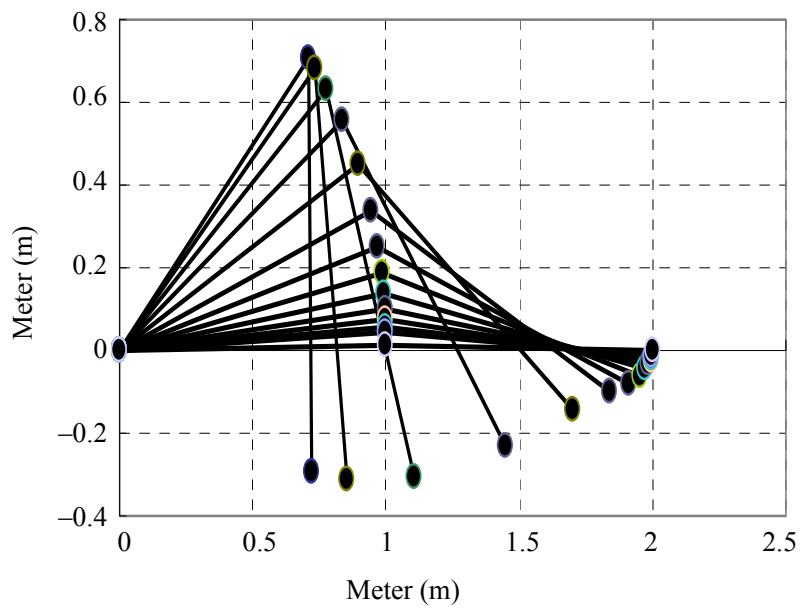

Figure 12. Trajectory of two-link manipulator for dragreduction control in case 2 .

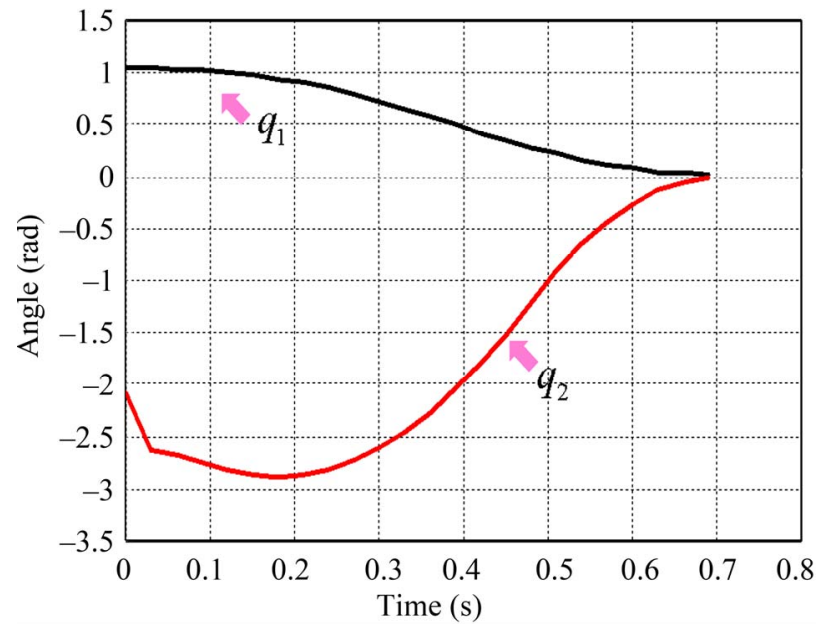

Figure 13. Time history of angles for time optimal control in case 2. 


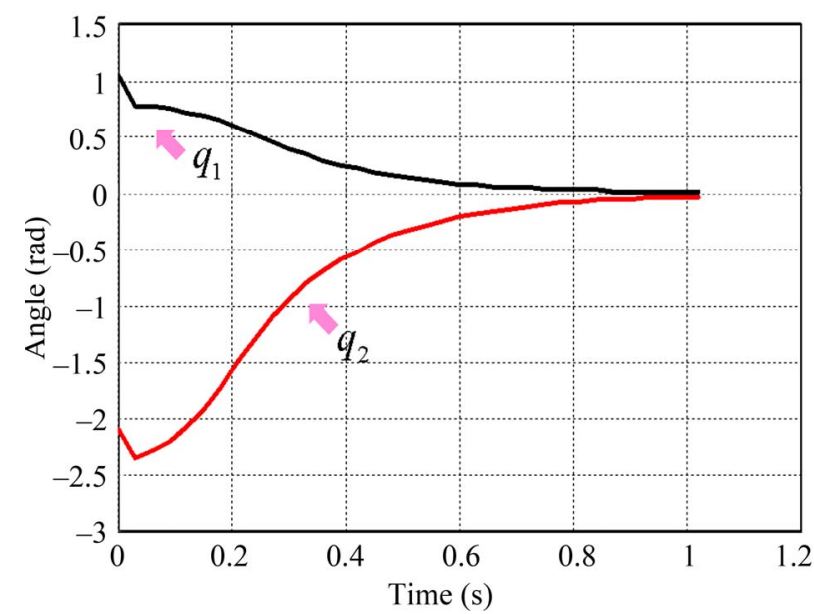

Figure 14. Time history of angles for drag reduction control in case 2.

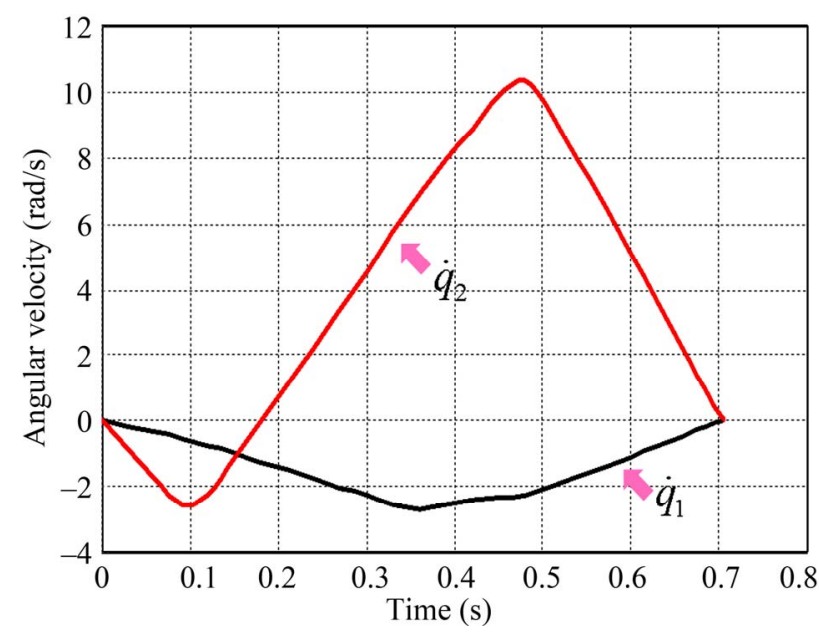

Figure 15. Time history of angular velocities for time optimal control in case 2 .

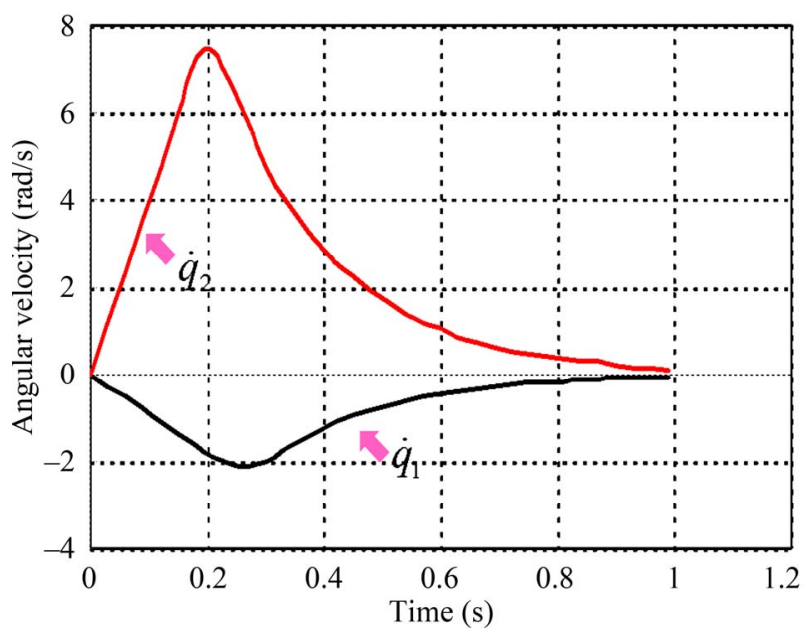

Figure 16. Time history of angular velocities for drag reduction control in case 2.

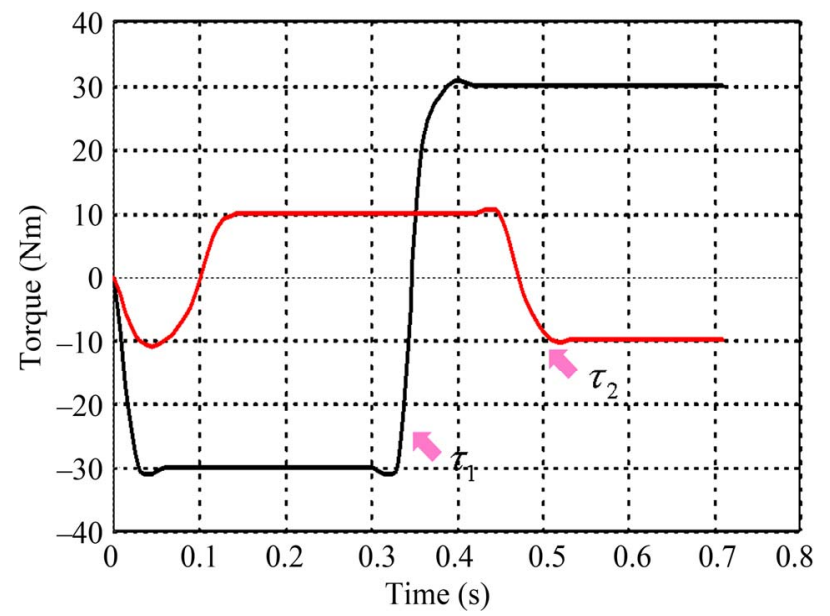

Figure 17. Time history of torques for time optimal control in case 2.

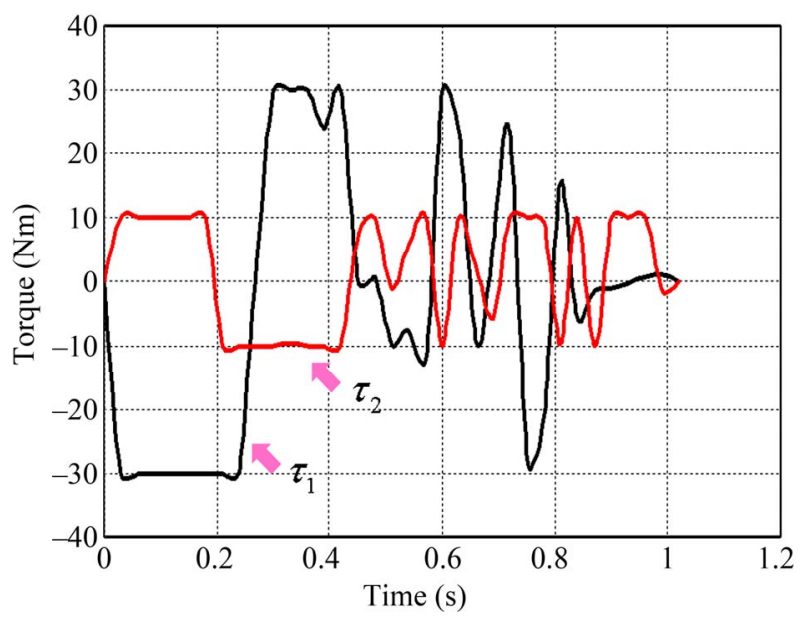

Figure 18. Time history of torques for drag reduction control in case 2.

The time histories of the torques are shown in the Figure 18. The clockwise torque 1 takes effect during approximately the first $0.25 \mathrm{~s}$. After $0.25 \mathrm{~s}$, the counterclockwise torque takes effect. The clockwise and counterclockwise torques act alternately. The clockwise torque acts on joint 2 during the first $0.2 s$. The counterclockwise torque takes effect from $0.2 s$ to $0.4 s$. After approximately $0.43 s$, the alternative torque acts on joint 2 .

\section{Conclusions}

We proposed the adjoint variable method for obtaining an optimal trajectory in order to decrease the fluid drag on a manipulator when the manipulator moves from its initial position to the target position. By considering hydrodynamic effects, we formulated the dynamics of an underwater robot manipulator. The cost function was defined as the fluid drag. By introducing the adjoint 
variable, we formulated the Lagrange function under certain constraints, which consisted of the target angle, target angular velocities, and robot manipulator dynamics equations. The gradient of the Lagrange function with respect to the torque was derived from the stationary condition. The algorithm was developed on the basis of the adjoint variable method. This algorithm can be sufficiently converged to the optimum value under the constraints, and it can determine an optimal trajectory to reduce the fluid drag under the constraints.

Simulation results showed that the performance can be enhanced for the control of an underwater manipulator. By using this approach, we can use the motion to reduce fluid drag. It may be important to note that significant performance enhancement is achieved by the motion of the manipulator.

\section{References}

[1] T. L. McLain, S. M. Rock, and M. J. Lee, "Experiments in the Coordinated Control of an Underwater Arm/Vehicle System," Autonomous Robots, Vol. 3, No. 2-3, 1996, pp. 213-232. doi:10.1007/BF00141156

[2] K. N. Leabourne and S. M. Rock, "Model Development of an Underwater Manipulator for Coordinated Arm-Vehicle Control," Proceedings of the OCEANS 98 Conference, Nice France, No. 2, 1998, pp. 941-946.

[3] J. Yuh, S. Zhao and P. M. Lee, "Application of Adaptive Disturbance Observer Control to an Underwater Manipulator," International Conference on Robotics and Automation, Vol. 4, 2001, pp. 3244-3249.

[4] S. Sagara, T. Tanikawa, M. Tamura and R. Katoh, "Experiments on a Floating Underwater Robot with a TwoLink Manipulator," Artificial Life and Robotics, Vol. 5, No. 4, 2001, pp. 215-219. doi:10.1007/BF02481505

[5] G. R. Vossoughi, A. Meghdari and H. Borhan, "Dynamic Modeling and Robust Control of an Underwater ROV Equipped with a Robotic Manipulator Arm,” 2004 Japan USA Symposium on Flexible Automation, Denver USA, 2004.

[6] K. Ioi and K. Itoh, "Modelling and Simulation of an Underwater Manipulator," Advanced Robotics, Vol. 4, No. 4, 1989, pp. 303-317. doi:10.1163/156855390X00152

[7] M. L. Nagurka and V. Yen "Optimal Design of Robotic
Manipulator Trajectories: A Nonlinear Programming Approach," Technical Report CMU-RI-TR-87-12, the Robotics Institute, Carnegie Mellon University, April, 1987.

[8] M. Žefran, "Review of the Literature on Time-Optimal Control of Robotic Manipulators," Technical Report MSCIS-94-30, University of Pennsylvania, Philadelphia, 1994.

[9] M. E. Kahn and B. Roth, "The Near Minimum-Time Control of Open-Loop Articulated Kinematic Chains," Journal of Dynamic Systems, Measurement and Control, Vol. 93, No. 3, 1971, pp. 164-172. doi: $10.1115 / 1.3426492$

[10] M. Vukobratović and M. Kirćanski, "A Method for Optimal Synthesis of Manipulation Robot Trajectories," Journal of Dynamic Systems, Measurement, and Control, Vol. 104, No. 2, 1982, pp. 188-193. doi:10.1115/1.3139695

[11] M. A. Townsend, "Optimal Trajectories and Controls for Systems of Coupled Rigid Bodies with Application of Biped Locomotion," Thesis (Ph.D.), University of Wisconsin Madison, Madison, 1971.

[12] Y. D. Lee and B. H. Lee, "Genetic Trajectory Planner for a Manipulator with Acceleration Parameterization," Journal of Universal Computer Science, Vol. 3, No. 9, 1997, pp. 1056-1073.

[13] D. Constantinescu and E. A. Croft, "Smooth and Time-Optimal Trajectory Planning for Industrial Manipulators Along Specified Paths," Journal of Robotic Systems, Vol. 17, No. 5, 2000, pp. 233-249. doi:10.1002/(SICI)1097-4563(200005)17:5<233::AID-R OB1>3.0.CO;2-Y

[14] E. Shintaku, "Minimum Energy Trajectory for an Underwater Manipulator and Its Simple Planning Method by Using a Genetic Algorithm," Advanced Robotics, Vol. 13, No. 6-13, 1999, pp. 115-138.

[15] Y. Uno, M. Kawato and R. Suzuki, "Formation and Control of Optimal Trajectory in Human Multijoint Arm Movement," Biological cybernetics, Vol. 61, No. 2, 1989, pp. 89-101. doi:10.1007/BF00204593

[16] J. Saleh, "Fluid Flow Handbook," McGraw Hill, New York, 2002.

[17] H. Kobayashi et al., "The Robot Control Actually," The Society of Instrument and Control Engineers, Tokyo, 1997. 


\section{Appendix A: Fluid Drag}

The underwater manipulator receives a reactive force when it moves to the target position. In this section, the fluid force on the manipulator is derived. In this study, the link is defined as a circular cylinder. The translational velocity with respect to the coordinates $\left(x_{0}, y_{0}, z_{0}\right)$ $(=(0,0,0))$ of link 1 is given by

$$
\boldsymbol{v}_{1}=\boldsymbol{v}_{0}+\boldsymbol{\omega}_{1} \times \boldsymbol{x}_{1}=\left[\begin{array}{l}
0 \\
0 \\
0
\end{array}\right]+\left[\begin{array}{c}
0 \\
0 \\
\dot{q}_{1}
\end{array}\right] \times\left[\begin{array}{c}
x_{1} \\
0 \\
0
\end{array}\right]=\left[\begin{array}{c}
0 \\
x_{1} \dot{q}_{1} \\
0
\end{array}\right]
$$

where the variable $v_{0}$ represents the velocity on the ground. In this study, the base is fixed at $\boldsymbol{v}_{0}=0$. The variable $\omega_{1}$ is the angular velocity vector in link 1 . The translational velocity in link 2 is given by

$$
\begin{aligned}
\boldsymbol{v}_{2} & =\boldsymbol{v}_{1}+\boldsymbol{\omega}_{2} \times \boldsymbol{x}_{2}=\left[\begin{array}{c}
0 \\
l_{1} \dot{q}_{1} \\
0
\end{array}\right]+\left[\begin{array}{c}
0 \\
0 \\
\dot{q}_{1}+\dot{q}_{2}
\end{array}\right] \times\left[\begin{array}{c}
x_{2} \\
0 \\
0
\end{array}\right] \\
& =\left[\begin{array}{c}
0 \\
l_{1} \dot{q}_{1}+x_{2}\left(\dot{q}_{1}+\dot{q}_{2}\right) \\
0
\end{array}\right]
\end{aligned}
$$

Using Equation (35), we obtain the fluid drag in link 1 as

$$
\frac{\rho}{2} C_{1} d_{1} \int_{0}^{l_{1}}\left(\boldsymbol{v}_{0}+\boldsymbol{\omega}_{1} \times \boldsymbol{x}_{1}\right)^{2} \cdot \operatorname{sgn}\left(x_{1} \dot{q}_{1}\right) \mathrm{d} x_{1}
$$

$$
=\frac{\rho}{2} C_{1} d_{1} \int_{0}^{l_{1}}\left[\begin{array}{c}
0 \\
x_{1}^{2} \dot{q}_{1}^{2} \cdot \operatorname{sgn}\left(x_{1} \dot{q}_{1}\right) \\
0
\end{array}\right] \mathrm{d} x_{1}
$$

The function $\operatorname{sgn}\left(x_{1} \dot{q}_{1}\right)$ always becomes the function $\operatorname{sgn}\left(\dot{q}_{1}\right)$ because $x_{1}>0$. Therefore, $\operatorname{sgn}\left(x_{1} \dot{q}_{1}\right)=$ $\operatorname{sgn}\left(\dot{q}_{1}\right)$ The integration in Equation (37) is not related to the function $\operatorname{sgn}\left(\dot{q}_{1}\right)$. Thus, Equation (37) becomes

$$
\begin{aligned}
{\left[\begin{array}{c}
0 \\
\frac{\rho}{2} C_{1} d_{1} \frac{1}{3} l_{1}^{3} \dot{q}_{1}^{2} \cdot \operatorname{sgn}\left(\dot{q}_{1}\right) \\
0
\end{array}\right] } & =\left[\begin{array}{c}
0 \\
\frac{\rho}{6} C_{1} d_{1} l_{1}^{3} \dot{q}_{1}^{2} \cdot \operatorname{sgn}\left(\dot{q}_{1}\right) \\
0
\end{array}\right] \\
& =\left[\begin{array}{c}
0 \\
D_{1} \\
0
\end{array}\right]=\boldsymbol{D}_{1}
\end{aligned}
$$

The fluid drag in link 2 is (see next page)

In this study, in order to simplify the formulation of the adjoint variable method, it is assumed to satisfy the inequality as

$$
l_{1} \dot{q}_{1}+l_{2}\left(\dot{q}_{1}+\dot{q}_{2}\right)>0
$$

Thus, Equation (39) becomes (see next page)

\section{Appendix B: Definition of Variables}

The variables used in this study can be defined as follows:

$$
A_{1}=B_{1} B_{2}-B_{2}^{2}-B_{3}^{2} \cos ^{2} q_{2}
$$

$$
\begin{aligned}
& \frac{\rho}{2} C_{2} d_{2} \int_{0}^{l_{2}}\left(\boldsymbol{v}_{1}+\boldsymbol{\omega}_{2} \times \boldsymbol{x}_{2}\right)^{2} \cdot \operatorname{sgn}\left\{l_{1} \dot{q}_{1}+x_{2}\left(\dot{q}_{1}+\dot{q}_{2}\right)\right\} \mathrm{d} x_{2}=\frac{\rho}{2} C_{2} d_{2} \int_{0}^{l_{2}}\left[\left\{l_{1} \dot{q}_{1}+x_{2}\left(\dot{q}_{1}+\dot{q}_{2}\right)\right\}^{2} \cdot \operatorname{sgn}\left\{l_{1} \dot{q}_{1}+x_{2}\left(\dot{q}_{1}+\dot{q}_{2}\right)\right\}\right] \mathrm{d} x_{2} \\
& =\frac{\rho}{2} C_{2} d_{2} \int_{0}^{l_{2}}\left[\left\{l_{1}^{2} \dot{q}_{1}^{2}+2 l_{1} \dot{q}_{1}\left(\dot{q}_{1}+\dot{q}_{2}\right) x_{2}+\left(\dot{q}_{1}+\dot{q}_{2}\right)^{2} x_{2}^{2}\right\} \cdot \operatorname{sgn}\left\{l_{1} \dot{q}_{1}+x_{2}\left(\dot{q}_{1}+\dot{q}_{2}\right)\right\}\right] \mathrm{d} x_{2} \\
& \frac{\rho}{2} C_{2} d_{2} \int_{0}^{l_{2}}\left(\boldsymbol{v}_{1}+\boldsymbol{\omega}_{2} \times \boldsymbol{x}_{2}\right)^{2} \cdot \operatorname{sgn}\left\{l_{1} \dot{q}_{1}+x_{2}\left(\dot{q}_{1}+\dot{q}_{2}\right)\right\} \mathrm{d} x_{2}=\left[\begin{array}{c}
0 \\
\frac{\rho}{2} C_{2} d_{2} l_{2}\left\{\left(l_{1}^{2}+l_{1} l_{2}+\frac{l_{2}^{2}}{3}\right) \dot{q}_{1}^{2}+\left(\frac{2}{3} l_{2}^{2}+l_{1} l_{2}\right) \dot{q}_{1} \dot{q}_{2}+\frac{l_{2}^{2}}{3} \dot{q}_{2}^{2}\right\} \\
0
\end{array}\right] \\
& =\left[\begin{array}{c}
0 \\
D_{2} \\
0
\end{array}\right]=\boldsymbol{D}_{2}
\end{aligned}
$$




$$
\begin{aligned}
& A_{2}=-B_{2}-B_{3} \cos q_{2} \\
& A_{3}=-B_{3} \dot{q}_{1}^{2} \sin q_{2} \\
& A_{4}=2 B_{3} \dot{q}_{1} \dot{q}_{2} \cos q_{2}+B_{3} \dot{q}_{2}^{2} \cos q_{2} \\
& A_{5}=2 B_{3} \dot{q}_{1} \dot{q}_{2} \sin q_{2}+B_{3} \dot{q}_{2}^{2} \sin q_{2} \\
& A_{6}=B_{1}+2 B_{3} \cos q_{2} \\
& A_{7}=2 B_{3} \dot{q}_{2} \sin q_{2} \\
& A_{8}=B_{3} \sin q_{2} \\
& A_{9}=B_{3} \cos q_{2} \\
& A_{10}=B_{3} \dot{q}_{1} \sin q_{2} \\
& A_{11}=B_{3} \dot{q}_{1}^{2} \cos q_{2} \\
& A_{12}=2 B_{3}\left(\dot{q}_{1}+\dot{q}_{2}\right) \sin q_{2} \\
& A_{13}=B_{4} \dot{q}_{1}^{2}+\left(B_{5} r_{2}+B_{5} l_{1} \cos q_{2}\right)\left(B_{6} \dot{q}_{1}^{2}+B_{7} \dot{q}_{1} \dot{q}_{2}\right. \\
& A_{14}=B_{5} r_{2}\left(B_{6} \dot{q}_{1}^{2}+B_{7} \dot{q}_{1} \dot{q}_{2}+B_{8} \dot{q}_{2}^{2}\right) \\
& A_{15}=-B_{5} \sin q_{2}\left(B_{6} \dot{q}_{1}^{2}+B_{7} \dot{q}_{1} \dot{q}_{2}+B_{8} \dot{q}_{2}^{2}\right) \\
& A_{16}=2 B_{6} \dot{q}_{1}+B_{7} \dot{q}_{2} \\
& A_{17}=2 B_{4} \dot{q}_{1}+\left(B_{5} r_{2}+B_{5} l_{1} \cos q_{2}\right)\left(2 B_{6} \dot{q}_{1}+B_{7} \dot{q}_{2}\right) \\
& A_{18}=B_{7} \dot{q}_{1}+2 B_{8} \dot{q}_{2} \\
& A_{19}=B_{5} r_{2}+B_{5} l_{1} \cos q_{2}
\end{aligned}
$$

where the variables $B_{1}-B_{8}$ are defined as follows:

$$
\begin{aligned}
B_{1} & =I_{z z 1}+I_{z z 2}+m_{2} l_{1}^{2} \\
& =\left(I_{z z g 1}+m_{1} r_{1}^{2}\right)+\left(I_{z z g 2}+m_{2} r_{2}^{2}\right)+m_{2} l_{1}^{2} \\
& =m_{1}\left(\frac{d_{1}^{2}}{16}+\frac{l_{1}^{2}}{12}\right)+m_{1} r_{1}^{2}+m_{2}\left(\frac{d_{2}^{2}}{16}+\frac{l_{2}^{2}}{12}\right)+m_{2} r_{2}^{2}+m_{2} l_{1}^{2} \\
B_{2} & =I_{z z 2}=I_{z z g 2}+m_{2} r_{2}^{2}=m_{2}\left(\frac{d_{2}^{2}}{16}+\frac{l_{2}^{2}}{12}\right)+m_{2} r_{2}^{2} \\
B_{3} & =m_{2} r_{2} l_{1} \\
B_{4} & =-\frac{\rho}{6} C_{1} d_{1} l_{1}^{3} \cdot \operatorname{sgn}\left(\dot{q}_{1}\right) \\
B_{5} & =-\frac{\rho}{2} C_{2} d_{2} l_{2} \\
B_{6} & =\left(l_{1}^{2}+l_{1} l_{2}+\frac{l_{2}^{2}}{3}\right)
\end{aligned}
$$

$$
\begin{aligned}
& B_{7}=\left(\frac{2}{3} l_{2}^{2}+l_{1} l_{2}\right) \\
& B_{8}=\frac{l_{2}^{2}}{3}
\end{aligned}
$$

\section{Appendix C: Robot Manipulator Dynamics}

The translational acceleration, $\boldsymbol{a}_{1}$, of the barycenter in link 1 is as

$$
\begin{aligned}
\boldsymbol{a}_{1} & =\dot{\boldsymbol{\omega}}_{1} \times \boldsymbol{r}_{1}+\boldsymbol{\omega}_{1} \times\left(\boldsymbol{\omega}_{1} \times \boldsymbol{r}_{1}\right) \\
& =\left[\begin{array}{c}
0 \\
0 \\
\ddot{q}_{1}
\end{array}\right] \times\left[\begin{array}{c}
r_{1} \\
0 \\
0
\end{array}\right]+\left[\begin{array}{c}
0 \\
0 \\
\dot{q}_{1}
\end{array}\right] \times\left(\left[\begin{array}{c}
0 \\
0 \\
\dot{q}_{1}
\end{array}\right] \times\left[\begin{array}{c}
r_{1} \\
0 \\
0
\end{array}\right]\right)=\left[\begin{array}{c}
-r_{1} \dot{q}_{1}^{2} \\
r_{1} \ddot{q}_{1} \\
0
\end{array}\right]
\end{aligned}
$$

The translational acceleration $\boldsymbol{a}_{2}$ of the barycenter in link 2 is given by

$$
\begin{aligned}
\boldsymbol{a}_{2}= & { }^{2} \boldsymbol{R}_{1} \boldsymbol{e}_{1}+\dot{\boldsymbol{\omega}}_{2} \times \boldsymbol{r}_{2}+\boldsymbol{\omega}_{2} \times\left(\boldsymbol{\omega}_{2} \times \boldsymbol{r}_{2}\right) \\
= & {\left[\begin{array}{ccc}
\cos q_{2} & \sin q_{2} & 0 \\
-\sin q_{2} & \cos q_{2} & 0 \\
0 & 0 & 1
\end{array}\right]\left[\begin{array}{c}
-l_{1} \dot{q}_{1}^{2} \\
l_{1} \ddot{q}_{1} \\
0
\end{array}\right]+\left[\begin{array}{c}
0 \\
0 \\
\ddot{q}_{1}+\ddot{q}_{2}
\end{array}\right] \times\left[\begin{array}{c}
r_{2} \\
0 \\
0
\end{array}\right] } \\
& +\left[\begin{array}{c}
0 \\
0 \\
\dot{q}_{1}+\dot{q}_{2}
\end{array}\right] \times\left(\left[\begin{array}{c}
0 \\
0 \\
\dot{q}_{1}+\dot{q}_{2}
\end{array}\right] \times\left[\begin{array}{c}
r_{2} \\
0 \\
0
\end{array}\right]\right) \\
= & {\left[\begin{array}{c}
-l_{1} \dot{q}_{1}^{2} \cos q_{2}+l_{1} \ddot{q}_{2} \sin q_{2}-r_{2}\left(\dot{q}_{1}+\dot{q}_{2}\right)^{2} \\
l_{1} \dot{q}_{1}^{2} \sin q_{2}+l_{1} \ddot{q}_{1} \cos q_{2}+r_{2}\left(\ddot{q}_{1}+\ddot{q}_{2}\right) \\
0
\end{array}\right] }
\end{aligned}
$$

The vector $\boldsymbol{e}_{i}$ represents the angular acceleration vector with respect to the tip of link $i$. The matrix ${ }^{2} \boldsymbol{R}_{1}$ represents the rotation matrix from coordinates $\left(x_{2}, y_{2}, z_{2}\right)$ to coordinates $\left(x_{1}, y_{1}, z_{1}\right)$. The translational forces $\boldsymbol{f}_{1}$ and $\boldsymbol{f}_{2}$ with respect to link 1 and link 2 are given by

$$
\begin{aligned}
& \boldsymbol{f}_{2}=m_{2} \boldsymbol{a}_{2}+\boldsymbol{D}_{2} \\
& \boldsymbol{f}_{1}=m_{1} \boldsymbol{a}_{1}+{ }^{1} \boldsymbol{R}_{2} \boldsymbol{f}_{2}+\boldsymbol{D}_{1}
\end{aligned}
$$

where the matrix ${ }^{1} R_{2}$ represents the rotation matrix from the coordinates $\left(x_{1}, y_{1}, z_{1}\right)$ to the coordinates $\left(x_{2}, y_{2}, z_{2}\right)$.

$$
{ }^{1} \boldsymbol{R}_{2}=\left[\begin{array}{ccc}
\cos q_{2} & -\sin q_{2} & 0 \\
\sin q_{2} & \cos q_{2} & 0 \\
0 & 0 & 1
\end{array}\right]
$$

In this study, the buoyancy and gravity are ignored. By equilibrant moment of the relation between link 1 and link 2 , it is derived as 


$$
\boldsymbol{n}_{2}=\boldsymbol{I}_{2} \boldsymbol{a}_{2}+\boldsymbol{\omega}_{2} \times\left(\mathbf{I}_{2} \boldsymbol{\omega}_{2}\right)-\boldsymbol{f}_{2} \times \boldsymbol{r}_{2}
$$

where the first and second terms on the right-hand side are

$$
\begin{gathered}
\boldsymbol{I}_{2} \boldsymbol{\alpha}_{2}=\left[\begin{array}{ccc}
I_{2 x} & 0 & 0 \\
0 & I_{2 y} & 0 \\
0 & 0 & I_{2 z}
\end{array}\right]\left[\begin{array}{c}
0 \\
0 \\
\ddot{q}_{1}+\ddot{q}_{2}
\end{array}\right]=\left[\begin{array}{c}
0 \\
0 \\
I_{2 z}\left(\ddot{q}_{1}+\ddot{q}_{2}\right)
\end{array}\right] \quad(75) \\
\boldsymbol{\omega}_{2} \times\left(\boldsymbol{I}_{2} \boldsymbol{\omega}_{2}\right)=\left[\begin{array}{c}
0 \\
0 \\
\dot{q}_{1}+\dot{q}_{2}
\end{array}\right] \times\left(\left[\begin{array}{ccc}
I_{2 x} & 0 & 0 \\
0 & I_{2 y} & 0 \\
0 & 0 & I_{2 z}
\end{array}\right]\left[\begin{array}{c}
0 \\
0 \\
\dot{q}_{1}+\dot{q}_{2}
\end{array}\right]\right)=\left[\begin{array}{l}
0 \\
0 \\
0
\end{array}\right]
\end{gathered}
$$

where the matrix $\boldsymbol{I}_{2}$ represents the inertia tensor of link 2 . The vector $\boldsymbol{\alpha}_{2}$ represents the acceleration of link 2 . The equilibrant moment at joint 1 is derived as

$$
\begin{aligned}
\boldsymbol{n}_{1}= & { }^{1} \boldsymbol{R}_{2} \boldsymbol{n}_{2}-\boldsymbol{f}_{1} \times \boldsymbol{r}_{1}+\left({ }^{1} \boldsymbol{R}_{2} \boldsymbol{f}_{2}\right)\left(\boldsymbol{r}_{1}-\boldsymbol{l}_{1}\right) \\
& +\boldsymbol{I}_{1} \boldsymbol{\alpha}_{1}+\boldsymbol{\omega}_{1} \times\left(\boldsymbol{I}_{1} \omega_{1}\right)
\end{aligned}
$$

where the matrix $\boldsymbol{I}_{1}$ represents the inertia tensor of link 1. The vector $\boldsymbol{\alpha}_{1}$ represents the acceleration of link 1 . With respect to the $z$ axis, moments $\boldsymbol{n}_{1}$ of the joint 1 and $\boldsymbol{n}_{2}$ of the joint 2 are defined as torques $\tau_{1}$ and $\tau_{2}$. Equation (24) is thus derived.

\section{Appendix D. Derivation of Adjoint Equations}

The adjoint equations are derived. The variables $q_{1}, q_{2}$, $\dot{q}_{1}, \dot{q}_{2}, \ddot{q}_{1}$, and $\ddot{q}_{2}$ are independent of each other. The partial differential equation of the Lagrange function with respect to $\dot{q}_{1}$ is given by

$$
\begin{aligned}
\frac{\partial L}{\partial \dot{q}_{1}}= & \varepsilon_{1}\left(\int_{0}^{t} 1 \mathrm{~d} t\right)\left(q_{1}-a\right)+2 \varepsilon_{3}\left(\dot{q}_{1}-c\right)-2 B_{4} \dot{q}_{1}-B_{5} A_{16} \\
& +\lambda_{1}\left(\frac{-2 A_{2} A_{10}+B_{2} A_{7}}{A_{1}}+\frac{A_{2} B_{5} r_{2} A_{16}+B_{2} A_{17}}{A_{1}}\right) \\
& +\lambda_{2}\left(\frac{A_{2} A_{7}-2 A_{10} A_{6}}{A_{1}}+\frac{A_{2} A_{17}+A_{6} B_{5} r_{2} A_{16}}{A_{1}}\right)
\end{aligned}
$$

The partial differential equation of the Lagrange function with respect to $\ddot{q}_{1}$ is given by

$$
\frac{\partial L}{\partial \ddot{q}_{1}}=-\lambda_{1}
$$

The partial differential equation of the Lagrange function with respect to $\dot{q}_{2}$ is given by

$$
\frac{\partial L}{\partial \dot{q}_{2}}=\varepsilon_{2}\left(\int_{0}^{t} 1 \mathrm{~d} t\right)\left(q_{2}-b\right)+2 \varepsilon_{4}\left(\dot{q}_{2}-d\right)-B_{5} A_{18}
$$

$$
\begin{aligned}
& +\lambda_{1}\left(\frac{B_{2} A_{12}+A_{2} B_{5} r_{2} A_{18}+B_{2} A_{18} A_{19}}{A_{1}}\right) \\
& +\lambda_{2}\left(\frac{A_{2} A_{12}+A_{2} A_{18} A_{19}+A_{6} B_{5} r_{2} A_{18}}{A_{1}}\right)
\end{aligned}
$$

The partial differential equation of the Lagrange function with respect to $\ddot{q}_{2}$ is given by

$$
\frac{\partial L}{\partial \ddot{q}_{2}}=-\lambda_{2}
$$

\section{Appendix E. Derivation of Adjoint Equations}

The time optimal control method is described in the literature [17]. The Hamiltonian formulation is applied in time optimal control. Using a Legendre transformation, the Hamiltonian formulation is derived from the Lagrangian formulation. The parameters are defined as follows:

$$
\begin{aligned}
& x_{1}=q_{1} \\
& x_{2}=q_{1} \\
& x_{3}=\dot{q}_{1} \\
& x_{4}=\dot{q}_{2}
\end{aligned}
$$

The state equation is defined as

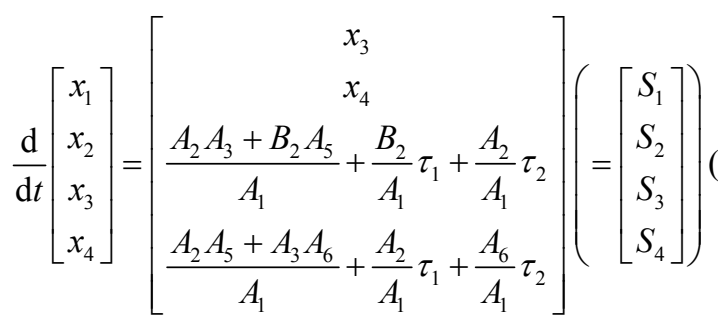

The cost function with respect to the time optimal control is given by

$$
J=\int_{0}^{t} L \mathrm{~d} t+N=\int_{0}^{t} 1 \mathrm{~d} t+N=t+N
$$

where the constraint $N$ represents the constraint condition and Equation (16). Using the adjoint variables $p_{i}(i=1,2$, $3,4)$, the Hamiltonian is defined as

$$
H=\boldsymbol{p}^{\mathrm{T}} \boldsymbol{S}-L
$$

where $L$ represents 1 of the integrand in Equation (87). The adjoint equation is given by

$$
\frac{\mathrm{d} \boldsymbol{p}}{\mathrm{d} t}=-\left\{\frac{\partial}{\partial \boldsymbol{x}}\left(\boldsymbol{p}^{\mathrm{T}} \boldsymbol{S}-L\right)\right\}^{\mathrm{T}}=-\left(\frac{\partial H}{\partial \boldsymbol{x}}\right)^{\mathrm{T}}
$$

The boundary condition of the adjoint equation is given by

$$
p\left(t_{f}\right)=-\left(\frac{\partial N\left(\mathbf{x}\left(t_{f}\right)\right)}{\partial \mathbf{x}\left(t_{f}\right)}\right)^{T}
$$


The Hamiltonian Equation (89) is

$$
H=-1+p_{1} x_{3}+p_{2} x_{4}+p_{3} S_{3}+p S_{4}
$$

Using Equation (89), the adjoint equation is given by

$$
\frac{\mathrm{d}}{\mathrm{d} t} p_{1}=0 \quad\left(=-\frac{\partial H}{\partial x_{1}}\right)
$$

$$
\begin{aligned}
\frac{\mathrm{d}}{\mathrm{d} t} p_{2}= & -\left\{\frac{B_{2} A_{4}+A_{3} A_{8}-A_{2} A_{11}}{A_{1}}-\frac{2\left(A_{2} A_{3}+B_{2} A_{5}\right) A_{8} A_{9}}{A_{1}^{2}}\right\} p_{3}-\left\{\frac{A_{2} A_{4}+A_{5} A_{8}-2 A_{3} A_{8}-A_{6} A_{11}}{A_{1}}-\frac{2\left(A_{2} A_{5}+A_{3} A_{6}\right) A_{8} A_{9}}{A_{1}^{2}}\right\} p_{4} \\
& -\left\{-\frac{2 B_{2} A_{8} A_{9}}{A_{1}^{2}}\right\} p_{3} \tau_{1}-\left\{\frac{A_{8}}{A_{1}}-\frac{2 A_{2} A_{8} A_{9}}{A_{1}^{2}}\right\} p_{3} \tau_{2}-\left\{\frac{A_{8}}{A_{1}}-\frac{2 A_{2} A_{8} A_{9}}{A_{1}^{2}}\right\} p_{4} \tau_{1}-\left\{-\frac{2 A_{8}}{A_{1}}-\frac{2 A_{6} A_{8} A_{9}}{A_{1}^{2}}\right\} p_{4} \tau_{2}\left(=-\frac{\partial H}{\partial x_{2}}\right)
\end{aligned}
$$

$\frac{\mathrm{d}}{\mathrm{d} t} p_{3}=-p_{1}-\frac{B_{2} A_{7}-2 A_{2} A_{10}}{A_{1}} p_{3}-\frac{A_{2} A_{7}-2 A_{6} A_{10}}{A_{1}} p_{4}\left(=-\frac{\partial H}{\partial x_{3}}\right)$

$\frac{\mathrm{d}}{\mathrm{d} t} p_{4}=-p_{2}-\frac{B_{2} A_{12}-B_{2} A_{2}}{A_{1}} p_{3}-\frac{A_{2} A_{12}-B_{2} A_{6}}{A_{1}} p_{4}\left(=-\frac{\partial H}{\partial x_{4}}\right)$

The constraint condition at the end time is given by

$$
\begin{aligned}
& p_{1}\left(t_{f}\right)=-2 \varepsilon_{1}\left(x_{1}\left(t_{f}\right)-a\right) \\
& p_{2}\left(t_{f}\right)=-2 \varepsilon_{2}\left(x_{2}\left(t_{f}\right)-b\right) \\
& p_{3}\left(t_{f}\right)=-2 \varepsilon_{3} x_{3}\left(t_{f}\right) \quad(c=0) \\
& p_{4}\left(t_{f}\right)=-2 \varepsilon_{4} x_{4}\left(t_{f}\right) \quad(d=0)
\end{aligned}
$$

The gradient is given by

$$
-\left[\begin{array}{l}
\frac{\partial H}{\partial \tau_{1}} \\
\frac{\partial H}{\partial \tau_{2}}
\end{array}\right]=-\left[\begin{array}{c}
p_{3} \frac{B_{2}}{A_{1}}+p_{4} \frac{A_{2}}{A_{1}} \\
p_{3} \frac{A_{2}}{A_{1}}+p_{4} \frac{A_{6}}{A_{1}}
\end{array}\right]
$$

